\title{
3D Organ Motion Prediction for MR-Guided High Intensity Focused Ultrasound
}

\author{
Patrik Arnold, Frank Preiswerk, Beat Fasel, Rares Salomir, Klaus Scheffler, \\ and Philippe C. Cattin
}

\begin{abstract}
Medical Image Analysis Center, University of Basel, 4000 Basel, Switzerland \{patrik.arnold,philippe.cattin\}@unibas.ch
\end{abstract}

\begin{abstract}
MR-guided High Intensity Focused Ultrasound is an emerging non-invasive technique capable of depositing sharply localised energy deep within the body, without affecting the surrounding tissues. This, however, implies exact knowledge of the target's position when treating mobile organs. In this paper we present an atlas-based prediction technique that trains an atlas from time-resolved 3D volumes using 4DMRI, capturing the full patient specific motion of the organ. Based on a breathing signal, the respiratory state of the organ is then tracked and used to predict the target's future position. To additionally compensate for the non-periodic slower organ drifts, the static motion atlas is combined with a population-based statistical exhalation drift model. The proposed method is validated on organ motion data of 12 healthy volunteers. Experiments estimating the future position of the entire liver result in an average prediction error of $1.1 \mathrm{~mm}$ over time intervals of up to 13 minutes.
\end{abstract}

\section{Introduction}

Respiratory organ motion is a complicating factor in the treatment of pathological tissue with MR-guided High Intensity Focused Ultrasound (MRgHIFU). Focused ultrasound has the unique capability to deposit sharply localised energy deep into the tissues, producing thermal ablation. Accurate spatial and rapid temporal beam spot focusing in the range of millimetres and within milliseconds, respectively, is reachable and hence increasing the demand of more exact knowledge about the organ's position. Accurate tracking of pathological tissue in mobile organs would not only increase patient safety, but also reduce the treatment time, as the gating window can be increased without sacrificing precision. Although the patient is located within the MR system during sonication, the scan-time is mainly required for the temperature feedback control of the HIFU system to determine the thermal dose given to a tumour. Non MR-based external surrogate markers would thus be ideal for the prediction of the respiratoryinduced organ motion. Several techniques have been proposed in the literature to handle respiratory organ motion. Existing approaches that ensure comprehensive target coverage with minimal damage to the surrounding tissue include the optimisation of safety margins, voluntary or forced breath-hold, respiratory 
gating or full tracking of the target. These methods were discussed in depth and compared in recent publications [12]. The holy grail of three-dimensional motion compensation in free-breathing awake patients is still out-of-reach, though. On the one hand, this goal could be reached by using ultrasound for real-time tracking [3]. On the other hand, Ries et al. 4] proposed only recently a real-time tracking method that observes the target on a $2 \mathrm{D}$ image plane combined with a perpendicular acquired pencil beam navigator, finally obtaining 3D information of the targets trajectories. The future target position is then estimated by a 3D Kalman filter. The method was tested in phantom experiments on human kidneys and in vivo with kidneys of ventilated pigs, both following a regular and stable breathing pattern. The tracking quality is evaluated by comparing temperature distribution obtained after $60 \mathrm{~s}$ of HIFU application with and without motion compensation, resulting in higher final temperatures in the target area with enabled motion compensation.

In this paper, we present a novel atlas-based respiratory motion prediction method for free breathing patients. In contrast to the state-of-the-art, the slower modes of non-periodic organ deformation, that occur in addition to the fitful respiratory motion and that are not detectable by external sensors, are compensated by means of a population-based statistical drift model. Although the proposed generic framework is applicable to any abdominal organ, e.g. the kidney, the prediction technique is evaluated on real 4DMRI motion data of the liver.

\section{Materials and Methods}

\subsection{Data Acquisition}

To learn the patient setup specific breathing characteristics and organ motion, 4DMRI 5] sequences of 12 healthy volunteers (6 female, 6 male, average age 31 , range 17-75) were acquired. During roughly one hour acquisition sessions, 1426 minutes of time-resolved motion data was captured. MR volumes consisting of 25-30 slices covering the right liver lobe with a voxel size of $1.4 \times 1.4 \times 4 \mathrm{~mm}^{3}$ and with a temporal resolution of 300-400 ms were obtained. The retrospectively reconstructed stacks cover the entire range of observed breathing depths. The vector fields describing the motion between the different respiratory states of the liver were estimated by means of 3D non-rigid registration [56] between the reconstructed volumes. In order to estimate the liver's future position we need to keep track of the current respiratory state on the basis of a breathing signal (surrogate marker). Regardless whether we measure the breathing signal by a breathing belt, by an optical chest wall tracker or by a pencil beam navigator placed on the diaphragm, the different respiratory states of the liver can reliable be tracked over a short period of time, as has been shown in [7. In this work, we extracted a pencil beam navigator by tracking a manually defined region (Fig. 1(a) on the navigator slice. The inferior-superior motion of the diaphragm was persistently tracked by template matching the dedicated region with all subsequent navigator frames throughout the acquisition sequence, providing one respiratory position per reconstructed volume (Fig. 1(b)). 
(a)

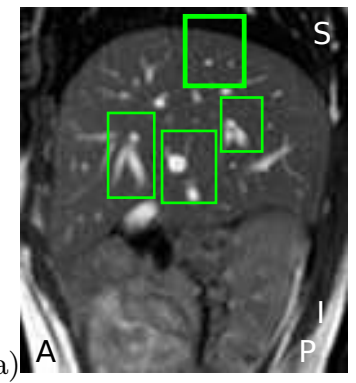

(b)

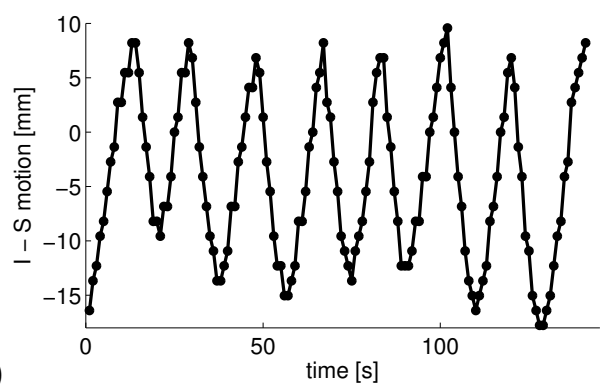

Fig. 1. (a) Sagittal view of a navigator slice with 4 marked regions used for slice stacking and drift compensation. The region indicated by the thicker frame is dedicated to track the diaphragm's position, providing the respiratory signal (b).

\subsection{Atlas Creation}

Using the 4D organ motion data of all 12 volunteers, we simulated a realistic MRgHIFU scenario. In particular, the first 7-13 minutes of 4DMRI scan time were used to build the motion atlas. This initial training time was long enough to cover all typical respiratory cycles. The remaining $4 \mathrm{D}$ motion data was used as ground truth to validate our prediction scheme. In order to keep correspondence between the acquisition of the atlas and the final treatment, the volunteers were asked not to move over the course of the entire sessions.

For each patient, a specific atlas and ground truth dataset is created, wherein both the breathing signal and corresponding $3 \mathrm{D}$ vector fields are stored pair-wise for each time step. An example of such an atlas is illustrated on the left side of Fig. 2 as well as the ground truth data for the validation on the right, both containing the respiratory signal and the associated organ displacement depicted by the black arrows between the reconstructed volumes. The breathing signal and the $3 \mathrm{D}$ vector fields describing the motion covering 150-400 breathing cycles or 1200-2000 time steps, respectively, serve as the atlas' database.

\subsection{Motion Prediction}

To readjust the treatment focus, any breathing-controlled tracking method must be able to estimate the target's position at some future time. This estimation must be based on measurements of the past breathing signal. Since our approach deals with a rather low sample rate, we use the atlas as combined breath and 3D motion look-up-table instead of an on-line learning based algorithm. However, only realistic, already seen motion patterns are being generated. Let $S$ be the respiratory signal given as the sequence $S=\left.s_{j}\right|_{j=1, \ldots, m}$, with the indices denoting the running time steps $t$. At a given point in time $j$, the prediction provides an estimate $s_{p}=s_{j+\Delta}^{\prime}$ of $S$ and of the corresponding 3D motion vector field $\mathbf{u}_{p}=\mathbf{u}_{j+\Delta}^{\prime}$, describing the future displacement of the organ for a later time point (Fig. 2). In the following experiments, we predicted $\Delta=1$ time step into the future. One time step corresponds to roughly $300 \mathrm{~ms}$, given by the 


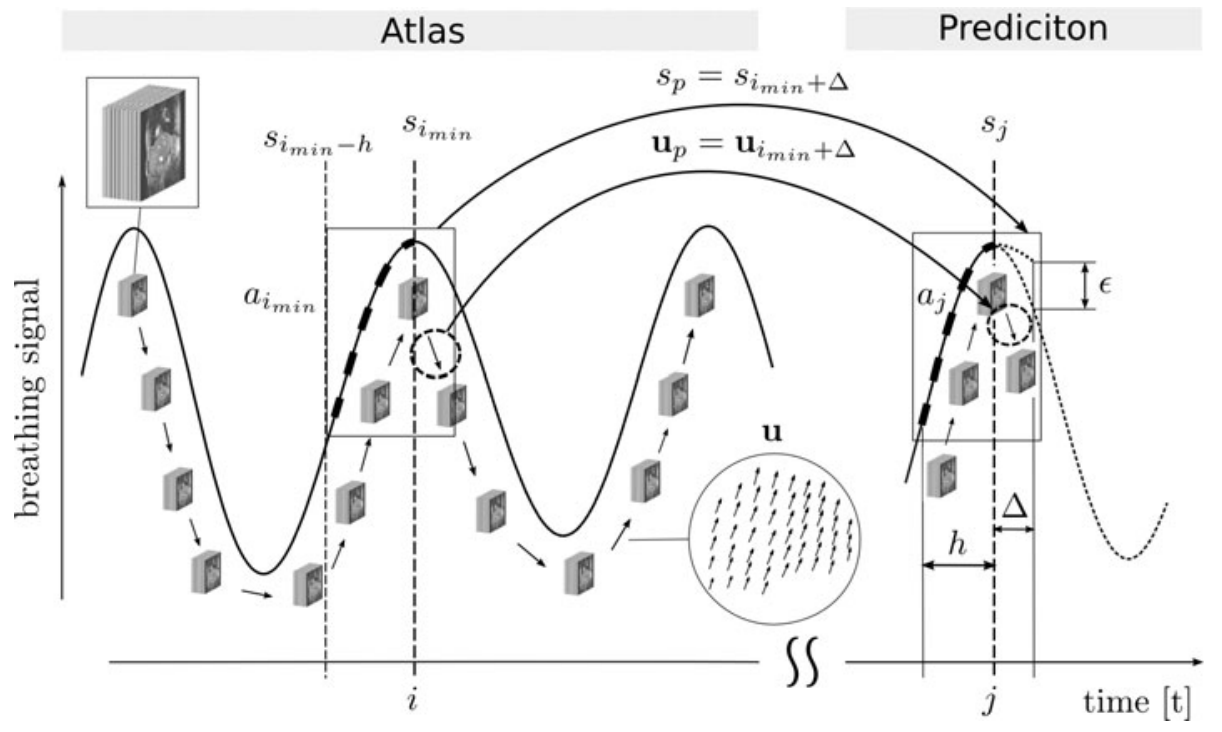

Fig. 2. Schematic illustration of the combined breath and motion atlas. Based on the signals history length $h$, the prediction yields the $3 \mathrm{D}$ displacement field $\mathbf{u}_{p}$ that estimates the organ's future position, $\Delta$ time steps ahead.

4 DMRI sequence. Note, that although the experiments are performed and validated on the temporal resolution of $3-4 \mathrm{~Hz}, \Delta$ can be chosen arbitrary. In that case, the breathing signals and the vector fields are interpolated, allowing any predictive time gap and smooth beam re-focusing. For the prediction, we propose using the last $h$ values of the breathing signals history denoted by the vector $\mathbf{a}_{j}=\left(s_{j-h}, \ldots, s_{j}\right)$. The reference signal $S_{r e f}$ basically serving as the atlas is represented by similar vectors $\mathbf{a}_{i}$ for prior time points $\mathbf{a}_{i}=\left(s_{i-h}, \ldots, s_{i}\right)$. The prediction of $S$ at the time point $j$ is chosen by finding the best match of the current breathing signal vector $\mathbf{a}_{j}$ within the reference signals from the atlas:

$$
i_{\min }=\arg \min _{i}\left|\mathbf{a}_{i}-\mathbf{a}_{j}\right| .
$$

The future run of $\mathbf{a}_{i_{\text {min }}}$ with minimum aberration from the history $\mathbf{a}_{j}$ is chosen to estimate the organ's prospective respiratory state and corresponding displacement field, $\Delta$ time steps ahead:

$$
s_{p}=s_{i_{m i n}+\Delta} \text { and } \quad \mathbf{u}_{p}=\mathbf{u}_{i_{m i n}+\Delta} .
$$

Finally, the task of predicting the organ's motion is handled by estimating the breathing signal's future evolution, yielding the well adapted displacement fields for the prediction. Since the algorithm is continuously adjusting to new input data, it can quickly adapt to the irregularity of the periods and amplitudes of the respiratory signal of a free breathing person. 


\subsection{Drift Compensation}

Besides the displacements of the liver caused by respiratory motion, additional deformation independent of the fitful breathing motion can occur within a few minutes. Since the proposed prediction method base on collecting the patientspecific liver motion during the initial training phase of a couple of minutes, these organ drifts can not be captured during the short acquisition of the atlas. These drifts can quickly invalidate the applicability of the static atlas with the consequence of increasing systematic prediction errors. However, during sonication within the MR system, the functionality of the scanner is used for temperature feedback of the HIFU device and therefore, a scan time intensive 3D drift tracking is hard to achieve. On the other side, measuring a one dimensional breathing signal only, tracking the inferior-superior motion of the diaphragm respectively, is not sensitive to drifts in the inferior part of the liver (Fig. 3(a)). We propose to acquire one update-navigator slice after every $60 \mathrm{~s}$ to capture the exhalation position of the liver based on the breathing signal. Comparing the displacements of the tracked regions with the regions on the actually acquired slice provides the needed information used for the correction of the previously acquired static atlas. In order to compensate these drifts, we introduce a population-based statistical drift model describing the inter-subject variations of exhalation positions in a shared shape-free coordinate system 8. Shape-free means, that only the relative differences to the first exhalation position of each subject, the drifts, respectively, are used for modelling. Thereby, we assume that the drift is independent of the respiratory motion and is similar for all subjects. From each subject, 200 exhalation positions $(m=11 \times 200)$ with $N=290$ corresponding points per liver $(n=3 N)$, placed on a $3 \mathrm{D}$ regular grid with a $15 \mathrm{~mm}$ resolution, are mean-free concatenated in a data matrix $\mathbf{X}=\left(\mathbf{x}_{1}, \mathbf{x}_{2}, \ldots, \mathbf{x}_{m}\right) \in$ $\mathbb{R}^{n \times m}$ with $\mathbf{x}_{k}=\mathbf{v}_{k}-\overline{\mathbf{v}}$ and sample mean $\overline{\mathbf{v}}=\frac{1}{m} \sum_{k=1}^{m} \mathbf{v}_{k}$. Applying Principal

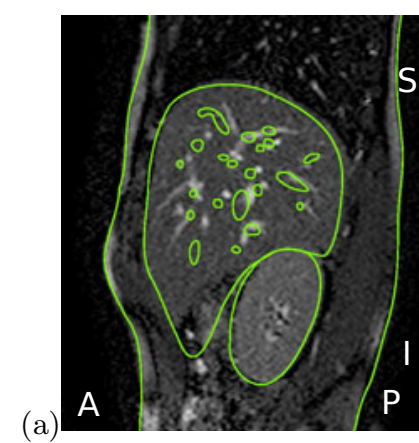

Fig. 3. (a)View of a sagittal placed navigator slice before (edges) and 20 minutes later after an exemplary drift displacement. The position of the diaphragm remains almost constant while the inferior part of the liver is drifting. (b) Ground truth motion field (black), prediction with static atlas (light grey) and with updated atlas (dark grey). 
Component Analysis on the data, the vectors $\mathbf{x}$ are defined by the coefficients $c_{k}$ and the eigenvectors $\mathbf{s}_{k}$ of $\mathbf{S}=\left(\mathbf{s}_{1}, \mathbf{s}_{2}, \ldots\right)$ of the covariance matrix of the data:

$$
\mathbf{x}=\sum_{k=1}^{m-1} c_{k} \sigma_{k} \mathbf{s}_{k}=\mathbf{S} \cdot \operatorname{diag}\left(\sigma_{k}\right) \mathbf{c} .
$$

Hereby, $\sigma_{k}$ are the standard deviations within the data along each eigenvector $\mathbf{s}_{k}$. As elaborated in [9], the full vector $\mathbf{x}$ can be found by an incomplete measurement $\mathbf{r} \in \mathbb{R}^{l}, l<n$ that minimises

$$
E(\mathbf{x})=\|\mathbf{L x}-\mathbf{r}\|^{2},
$$

where $\mathbf{L}$ represents a subspace mapping $\mathbf{L}: \mathbb{R}^{n} \mapsto \mathbb{R}^{l}$ such that $\mathbf{r}=\mathbf{L x}$. The reduced version of $\mathbf{S}$ can be written as $\mathbf{Q}=\mathbf{L S} \cdot \operatorname{diag}\left(\sigma_{k}\right) \in \mathbb{R}^{l \times m-1}$, yielding eigenvectors of the form $\mathbf{q}_{k}=\sigma_{k} \mathbf{L} \mathbf{s}_{k} \in \mathbb{R}^{l}$. The most probable organ deformation $\mathbf{v}$ given the incomplete measurements $\mathbf{r}$ is then

$$
\mathbf{v}=\mathbf{S} \cdot \operatorname{diag}\left(\sigma_{k}\right) \mathbf{c}+\overline{\mathbf{v}}, \text { where } \mathbf{c}=\mathbf{Q}^{+} \mathbf{r} .
$$

Hereby $\mathbf{Q}^{+}$is the pseudoinverse of $\mathbf{Q}$. The vector $\mathbf{r}$ describes the relative differences from a few grid points at the beginning of the data acquisition to the actual exhalation position. These displacements are captured again by template matching the defined regions (Fig. 1(a) with the update-navigator slice, measuring the distinct distances between the matching regions. Tracking 4 individual regions enables the detection of non-rigid deformations. As the centers of the templates may rarely coincide with the grid points of the model, the shifts of the templates have been adopted to the 3 closest points of the grid (12 out of 290 points), used as inputs for the drift model. The prediction from the static atlas $\mathbf{u}_{p}$ (Eq. 2) is updated by the non-rigid correction field (Fig. 3(b) provided by Eq. (5):

$$
\hat{\mathbf{u}}_{p}=\mathbf{u}_{p}+\mathbf{v}
$$

\section{Results}

\subsection{Motion Prediction without Drift Compensation}

In a first approach we evaluated the capability of organ motion compensation by means of a static atlas without drift compensation. Based on the data mentioned in Sec. 2.2 motion prediction experiments were performed on 4DMRI datasets of all 12 volunteers. The parameter $h$ introduced in Sec. 2.3 was optimised and found to work best for $h=3$ time steps $(\approx 1 \mathrm{~s})$. The prediction experiments were evaluated for all subjects, covering 75-200 full respiratory breathing cycles. The error in prediction for $\Delta=1$ time step $(\approx 300 \mathrm{~ms}$ ) was calculated point-wise over all grid points and time steps. The results are plotted in Fig. 4(a) characterised by the median, 5 th and 95 th percentiles. The dashed line is set to $2 \mathrm{~mm}$, marking an acceptable precision limit for HIFU treatments [10]. The impact of the liver's 
(a)
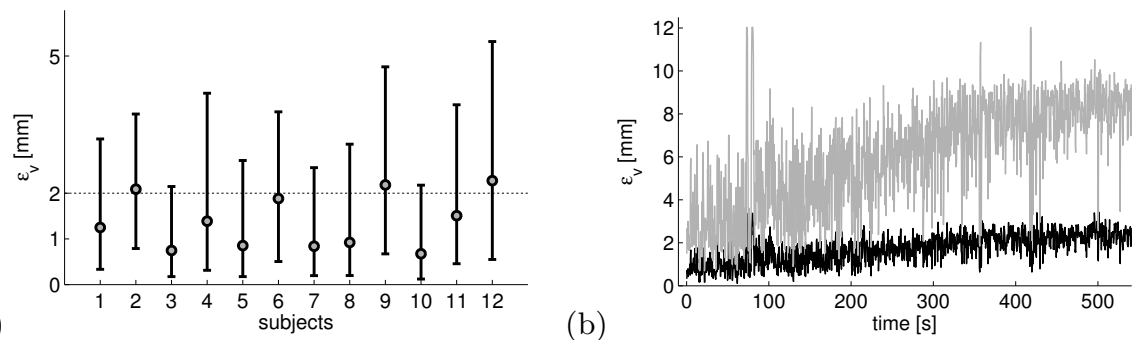

Fig. 4. (a) Resulting deviations between predicted and ground truth liver motions for 12 different subject over time intervals up to 13 minutes. Error bars around the median show the 5th and 95th percentile deviation. (b) Mean (black) and maximum error (grey) of motion prediction based on the static atlas for the drifting liver of subject 4 .

drift is clearly visible in Fig. 4(b), when monitoring the prediction performance over several minutes. The average error over all subjects is $1.6 \mathrm{~mm}$.

\subsection{Motion Prediction with Drift Compensation}

With equal settings as in Sec. 3.1, the same experiments but with drift compensation as elaborated in Sec. 2.4 were realised (Fig. 5(a)). The statistical drift models were built from 11 of 12 livers in leave-one-out experiments. Although the residual MR time during HIFU treatment is rather sparse, we allowed the acquisition of one 2D navigator slice every $60 \mathrm{~s}$, capturing the actual exhalation position. This time interval is based on the maximal observed drifting speed of $0.5 \mathrm{~mm} / \mathrm{min}$. Following Eq. (5) and (6), the most probable drift deformation of the left-out liver is provided by the model and used as a drift-update of the previously acquired static atlas. Taking the drift into account, the prediction performance remains constant over time as shown in Fig. 5(b), The error averaged over all subjects improved by $30 \%$ to $1.1 \mathrm{~mm}$, with a notable impact for

(a)

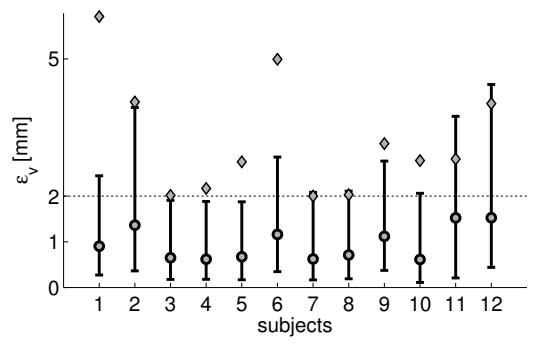

(b)

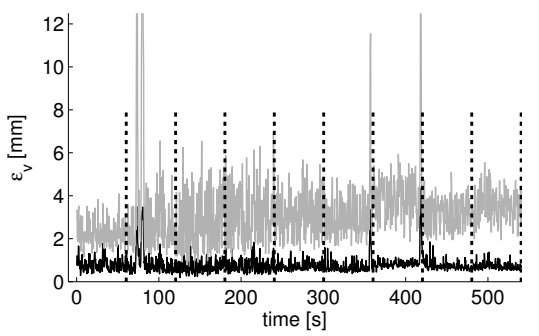

Fig. 5. (a) Residual error of motion prediction with drift compensation and median error without any motion compensation $(\diamond)$. (b) Mean (black) and maximum error (grey) of the prediction with drift compensation every $60 \mathrm{~s}$ (black-dotted lines). 
the subjects 4,9 and 12 . Without any motion compensation, the mean prediction error would be $4.7 \mathrm{~mm}$.

\section{Discussion and Outlook}

Despite frequently occurring organ drifts, our proposed method proved to be reliable enough for the application in MRgHIFU systems. Using the $\mathrm{R}$ software package (Version 2.11.1), we used the Kolmogorof-Smirnov test to test the mean errors of both experiments for normality. Assuming a significance level of 0.05, the t-tests showed that the statistical drift model significantly improved the prediction accuracy $(\mathrm{p}<0.05)$. By replacing the pencil beam navigator with a faster low lag signal, such as the breathing belt or a spirometer, the prediction quality should further improve, as the time span the system has to predict into the future decreases.

Acknowledgments. This work has been supported by the research network of the Swiss National Science Foundation-Project Nr. CR32I3_125499.

\section{References}

1. Keall, P., Mageras, G., Balter, J., Emery, R., Forster, K., Jliang, S., Kapatoes, J., Low, D., Murphy, M., Ramsey, B.M.C., Herk, M.V., Vedam, S., Wong, J., Yorke, E.: The management of respiratory motion in radiation oncology report of AAPM task group. Med. Phys. 33, 3874-3900 (2006)

2. Webb, S.: Motion effects in (intensity modulated) radiation therapy. Phys. Med. Biol. 51, R403-R425 (2006)

3. Pernot, M., Tanter, M., Fink, M.: 3-D real-time motion correction in high-intensity focused ultrasound therapy. Ultrasound in Medicine and Biology 30(9), 1239-1249 (2004)

4. Ries, M., de Senneville, B.D., Roujol, S., Berber, Y., Quesson, B., Moonen, C.: Real-time 3D target tracking in mri guided focused ultrasound ablations in moving tissues. Magnetic Resonance in Medicine 64, 1704-1712 (2010)

5. von Siebenthal, M., Szekely, G., Gamper, U., Boesiger, P., Lomax, A., Cattin, P.: $4 \mathrm{D}$ MR imaging of respiratory organ motion and its variability. Phys. in Med. Biol. 52, 1547-1564 (2007)

6. Rueckert, D., Sonoda, L.I., Hayes, C., Hill, D.L.G., Leach, M.O., Hawkes, D.J.: Nonrigid registration using free-form deformations: Application to breast MR images. Transactions on Medical Imaging 18, 712-721 (1999)

7. von Siebenthal, M., Szekely, G., Lomax, A.J., Cattin, P.C.: Systematic errors in respiratory gating due to intrafraction deformations of the liver. Med. Phys. 34, 3620-3629 (2007)

8. von Siebenthal, M., Székely, G., Lomax, A., Cattin, P.C.: Inter-subject modelling of liver deformation during radiation therapy. In: Ayache, N., Ourselin, S., Maeder, A. (eds.) MICCAI 2007, Part I. LNCS, vol. 4791, pp. 659-666. Springer, Heidelberg (2007)

9. Blanz, V., Vetter, T.: Reconstructing the complete 3D shape of faces from partial information. Informationstechnik und Technische Informatik 44, 295-302 (2002)

10. Vedam, S., Kini, V.R., Keall, P.J., Ramakrishnan, V., Mostafavi, H., Mohan, R.: Quantifying the predictability of diaphragm motion during respiration with a noninvasive external marker. Med. Phys. 30, 505-513 (2003) 